Revista Brasileira de Agricultura Irrigada v.8, no. 2, p. 171 - 187, 2014

ISSN 1982-7679 (On-line)

Fortaleza, CE, INOVAGRI - http://www.inovagri.org.br

DOI: $10.7127 /$ rbai.v8n200229

Protocolo 229/14 - 04/10/2013 Aprovado em 18/03/2014

\title{
RESPOSTA PRODUTIVA DO ARROZ (Oryza sativa L.) CULTIVADO EM DIFERENTES AGRO-ECOSSISTEMAS COM MANEJOS DE ÁGUA SEM SATURAÇÃO DO SOLO
}

\author{
Roberto Paulo Marano ${ }^{1}$, Marcos Vinicius Folegatti ${ }^{2}$, Silvia Imhoff ${ }^{3}$
}

\section{RESUMO}

Na região central de Santa Fe, Argentina, são cultivadas variedades de arroz anaeróbico com inundação continua em diferentes solos e condições hidrológicas. Objetivou-se avaliar a resposta produtiva do arroz em diferentes agro-ecossistemas: várzea (2011-12) e terraço (2012-13), com manejos alternativos de água: saturação com inundação intermitente (SIN) e não saturação com aspersão (NSA). O desenho estatístico foi parcela principal (agroecossistemas) e sub-parcelas (tratamentos) com repetições aleatórias. Em ambos os agroecossistemas foi utilizado o cultivar Puitá INTA, clearfield, desenvolvido para condições anaeróbicas. A colheita foi manual, separando grão da palha para estimar produtividade e suas componentes. A duração do ciclo e os estádios fenológicos não diferiram entre agroecossistemas. Foram determinadas diferenças significativas no manejo NSA do agroecossistema várzea em relação ao resto dos tratamentos para às variáveis produtividade, número de panículas e fertilidade das espiguetas, no entanto a variável massa de 1000 grãos foi muito estável. As diferenças na produtividade do tratamento NSA do agro-ecossistema várzea foram devidas às baixas taxas de infiltração e condutividade hidráulica, o que imposibilitou que as irrigações feitas durante o estádio reprodutivo ficassem efetivamente disponíveis para as plantas. Decorrentemente houve maior mortandade de panículas e esterilidade das espiguetas. $\mathrm{O}$ manejo SIN permitiu obter altas produtividades em ambos os agro-ecossistemas $\left(10,4 \mathrm{Mg} \mathrm{ha}^{-1}\right)$, resultando uma alternativa à inundação continua. No caso do agro-ecossistema terraço, o manejo com aspersão foi muito satisfatório e permitiu constatar que uma variedade anaeróbica pode ter alta produtividade em condições de solo não saturado.

Palavras-chave: arroz anaeróbico; aspersão; inundação intermitente

\footnotetext{
${ }^{1}$ Doutorando Programa Irrigação e Drenagem ESALQ-USP, Magíster Scientiae Riego y Drenaje, Pesquisador Facultad de Ciencias Agrarias (FCA), Universidad Nacional del Litoral (UNL), Kreder 2805, Esperanza, CP 3085, Argentina- email: rpmarano@gmail.com

${ }^{2}$ Doutor, Pesquisador da ESALQ, USP, Piracicaba, SP, Brasil Av. Pádua Dias, 11 - Piracicaba - SP, CEP 13418900. email: mvfolega@usp.br

${ }^{3}$ Doutora, Pesquisadora da FCA UNL, Argentina, Kreder 2805, Esperanza, CP 3085, Argentina- email: simhoff@fca.unl.edu.ar
} 


\title{
YIELD RESPONSE OF RICE (Oryza sativa L.) GROWN IN DIFFERENT AGROECOSYSTEMS WITH MANAGEMENTS OF WATER WITHOUT SOIL SATURATION
}

\begin{abstract}
In the central region of Santa Fe, Argentina, are cultivated varieties of anaerobic rice with flooding continues in different soils and hydrological conditions. This work aimed to evaluate the yield response of rice with two water management: saturation with intermittent flooding (SIF) and not saturation with sprinkle irrigation (NSS); agroecosystem lowland (2011-12) and agroecosystem upland (2012-13). The statistical design was main plot (agroecosystem) and subplots (treatments) with random repetitions. In both agroecosystems the cultivar Puita INTA, clearfield, developed for anaerobic conditions, was used. The harvesting was manual, separating grain from straw to estimate yield and components. The cycle duration and growth stages did not differ between agroecosystems. Were determined significant differences between NSS management of lowland agroecosystem in relation to the rest of the treatments for yield, number of panicles and spikelet fertility variables, however the variable mass of 1000 grains was very stable. The differences in the yield of NSS management of lowland agroecosystem were due to the small rates of infiltration and hydraulic conductivity, which not allowed that the irrigations made at the flowering stage, remain available to plants. As a consequence, a greater death of panicles and spikelets sterility was verified. The SIF management enable to obtain high yields in both agro-ecosystems $\left(10.4 \mathrm{Mg} \mathrm{ha}^{-1}\right)$, resulting an alternative to flood continues. In the agroecosystem upland, the sprinkler management was very satisfactory and allowed to establish that an anaerobic variety can have high yield under conditions of unsaturated soil.
\end{abstract}

Keywords: anaerobic rice-sprinkle irrigation-intermittent flooding

\section{INTRODUÇÃO}

A agricultura é essencial para fornecer alimentos à população mundial, estimada em 7.200 milhões (2013), com previsão de aumentar 1.000 milhões em 2025 até alcançar 9.600 milhões em 2050 (UNFPA, 2013). O maior crescimento da população ocorre em países em desenvolvimento, onde os cereais são a base da alimentação, sendo o arroz um dos principais produzidos (FAOSTAT, 2013). Argentina produziu 1,397 milhões de toneladas de arroz casca na safra 2012-13, com 222.695 ha colhidas
(ACPA, 2013) e produtividade média de 6,3 Mg $\mathrm{ha}^{-1}$. As províncias produtoras encontram-se na região Litoral, sendo Corrientes e Entre Rios as principais, seguido de Santa Fe. Nessa província a produção de arroz é promissora, com acréscimo de até $100 \%$ da área cultivada na última década (MARANO et al., 2013). Para isso conjugou-se a disponibilidade de terra, e fundamentalmente a de água, cuja qualidade e quantidade são excelentes e de fácil extração a partir de braços do rio Paraná.

Boa parte dos solos desta Região que foram incorporados à produção de arroz tem 
limitantes, tanto físicas (textura, adensamento), quanto químicas, (pH, sódio) (PILATTI et al., 2003) e constituem agro-ecossistemas diferentes. A inclusão desse termo obedece à necessidade de involucrar um sistema real de produção agropecuária no ecossistema, neste caso arroz em combinação com gado, para assim poder caracterizá-lo em relação ao ecossistema (PILATTI et al., 2011).

Neste período, o arroz dispõe de 2.500 $\mathrm{mm}$, onde as precipitações pluviométricas (PP) representam entre 400 e $600 \mathrm{~mm}$ e o restante é aportado pela irrigação (MARANO et al., 2013), sendo o método tradicional a inundação contínua (Ic), com uma lâmina armazenada na superfície do solo entre 8 a $10 \mathrm{~cm}$, porquanto as $\mathrm{PP}$ que ocorrem durante o ciclo da cultura, em geral, não são aproveitadas e escorrem para os drenagems.

Os cultivares de arroz utilizados em Santa Fe são adaptados para permanecer submergidos durante quase todo o ciclo, no entanto para o aumento da eficiência de irrigação, numerosos autores pesquisaram manejos alternativos à Ic: por exemplo, saturação com inundação intermitente (SIN, BELDER et al., 2004; BOUMAN et al., 2006; MEZZOMO, 2009) e também não saturação com aspersão (NSA, STONE; SILVEIRA, 2004; TOESCHER et al., 1997; CRUSCIOL et al., 2006). O presente trabalho teve como objetivo avaliar a resposta produtiva aos manejos SIN e NSA como alternativos para o arroz irrigado, em agroecossistemas contrastantes.

\section{MATERIAL E MÉTODOS}

O clima da região de estudo, de acordo com a classificação de Thornthwaite, é subúmido úmido (C2), mesotermal (MOSCONI et al., 1981). As máximas precipitações ocorrem durante o verão, enquanto que as mínimas ocorrem no inverno, sendo que entre 1982 e 2000, a precipitação média anual foi de $1.063 \mathrm{~mm}$. A água para irrigação é extraída do rio San Javier, braço do Paraná (condutividade elétrica de $0,115 \mathrm{dSm}^{-1}$ ) e conduzida através de canais de terra (sem revestimento) até as parcelas (CARNEVALE et al., 2011).

Foram selecionados os agro-ecosisstemas Várzea $(\mathrm{Vz})$ e Terraço $(\mathrm{Tç})$. O primeiro ocupa uma superfície em torno de 120.000 ha estendidas de leste a oeste e corresponde a áreas com relevo muito plano e declividade média entre 0,0005 a $0,0001 \mathrm{~m} \mathrm{~m}^{-1}$ (PILATTI et al., 2003). O escoamento superficial ocorre predominantemente pelo gradiente hidráulico antes que o topográfico, os cursos de água naturais estão longe e o lençol freático encontrase perto da superfície do terreno todo ano (MARANO et al., 2013). O solo predominante é Planossolo nátrico (PlaNa, Tabela 1), textura fina, altos conteúdos de $\mathrm{Na}^{+}$, baixa capacidade de infiltração e drenagem impedido (PILATTI et al., 2003). Neste agro-ecossistema foi desenvolvido o experimento na safra 2011-12 em San Javier, Santa Fe, Argentina (30 30’ 42” Sul 


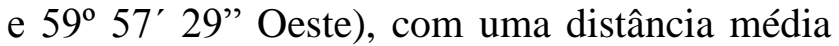
do rio San Javier de $15 \mathrm{~km}$ (Figura 1).

Tabela 1. Caracterização química dos solos das áreas experimentais

\begin{tabular}{|c|c|c|c|c|c|c|}
\hline \multirow{2}{*}{ Variável } & \multirow{2}{*}{ Unidade } & \multicolumn{2}{|c|}{$\mathrm{PlaNa}$} & \multicolumn{2}{|c|}{$\operatorname{Reg} \mathrm{Ab}$} & \multirow{2}{*}{$\begin{array}{l}\text { Níveis ótimos } \\
\text { para alta } \\
\text { produtividade } \\
\text { de arroz (1) }\end{array}$} \\
\hline & & $0-0,11$ & $0,11-0,25$ & $0-0,19$ & $0,19-0,4$ & \\
\hline MO & $(\%)$ & 3,10 & 1,50 & 2,23 & 1,13 & $2,5-8$ \\
\hline $\mathrm{CO}$ & $(\%)$ & 1,80 & 0,87 & 1,31 & 0,66 & - \\
\hline $\mathrm{Nt}$ & $(\%)$ & 0,166 & 0,097 & 0,12 & 0,08 & - \\
\hline $\mathrm{CO} / \mathrm{N}$ & - & 11 & 9 & 11 & 8 & - \\
\hline $\mathrm{P}$ & (ppm) & 10 & 7 & 50,00 & 46,67 & - \\
\hline $\mathrm{pH}$ & - & 6,1 & 7,2 & 6,07 & 6,23 & $4,8-6,5$ \\
\hline CEe & $\left(\mathrm{dS} \mathrm{m} \mathrm{m}^{-1}\right)$ & 0,9 & 0,6 & 0,30 & 0,23 & $<1,5$ \\
\hline $\mathrm{Ca}^{2+}$ & $\left(\mathrm{cmol}_{\mathrm{c}} \mathrm{kg}^{-1}\right)$ & 4,8 & 4,3 & 6,17 & 6,97 & $5-30$ \\
\hline $\mathrm{Mg}^{2+}$ & $\left(\mathrm{cmol}_{\mathrm{c}} \mathrm{kg}^{-1}\right)$ & 5,7 & 1,1 & 1,77 & 1,47 & $1,5-6,5$ \\
\hline $\mathrm{Na}^{+}$ & $\left(\mathrm{cmol}_{\mathrm{c}} \mathrm{kg}^{-1}\right)$ & 0,8 & 1,4 & 0,53 & 0,53 & - \\
\hline $\mathrm{K}^{+}$ & $\left(\mathrm{cmol}_{\mathrm{c}} \mathrm{kg}^{-1}\right)$ & 0,4 & 0,3 & 0,67 & 0,57 & $0,3-1,2$ \\
\hline CIC & $\left(\mathrm{cmol}_{\mathrm{c}} \mathrm{kg}^{-1}\right)$ & 9,2 & 8,8 & 15,97 & 15,30 & - \\
\hline PST & $(\%)$ & 8,7 & 15,9 & 3,4 & 3,6 & $<8$ \\
\hline $\begin{array}{c}\text { Saturação } \\
\mathrm{Ca}^{2+}\end{array}$ & $(\%)$ & 52 & 49 & 39,0 & 46,3 & $30-80$ \\
\hline $\begin{array}{c}\text { Saturação } \\
\mathrm{Mg}^{2+}\end{array}$ & $(\%)$ & 62 & 13 & 11,5 & 9,9 & $8-22$ \\
\hline $\begin{array}{c}\text { Saturação } \\
\mathrm{K}^{+}\end{array}$ & $(\%)$ & 4,3 & 3,4 & 4,3 & 3,8 & $1-4$ \\
\hline
\end{tabular}

Nota - (1) Segundo Quintero e Figueroa (2008)

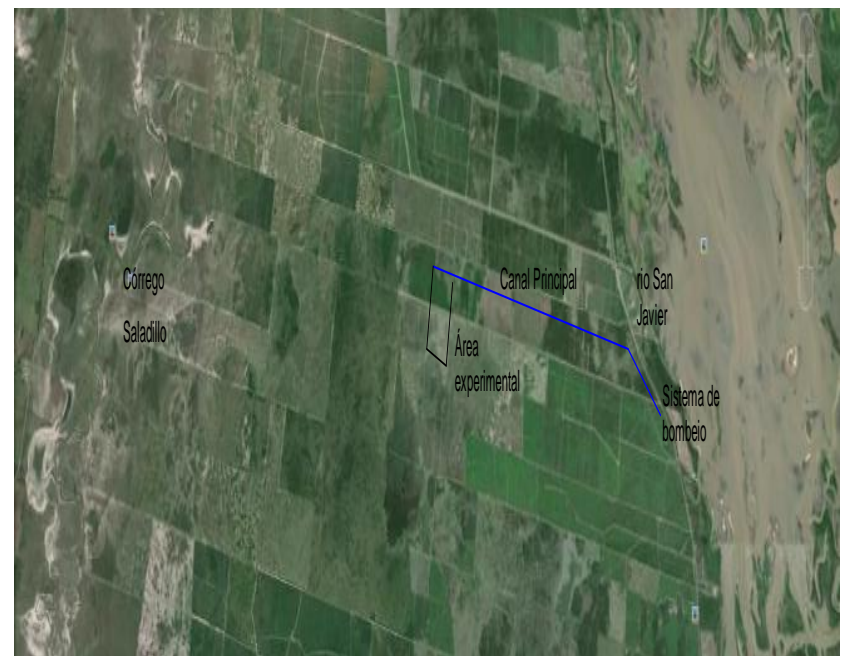

Figura 1. Agro-ecossistema Várzea $(\mathrm{Vz})$ com área experimental com solo PlaNa, Río San Javier a leste e córrego Saladillo a oeste. Fonte: Google Earth, ingresso 10-01-14
O agro-ecossistema Tç é um área adjacente à beira do Paraná que ocupa uma superfície de 37.000 ha, estendida de norte a sul. Pertenece a um campo de dunas longitudinales formadas pelos ventos (RAMONELL et al., 2013). O solo tem melhor drenagem, sem presença de $\mathrm{Na}^{+}$, declividade também plana, porém, de maior magnitude (média de $0,001 \mathrm{~m} \mathrm{~m}^{-1}$ ). A presença do rio San Javier nas imediações significa uma via de saída rápida tanto para o fluxo superficial como do lençol freático (Figura 2). Outra diferença com o agro-ecossistema $\mathrm{Vz}$ radica na possibilidade de fazer rotação agrícola (trigosoja-milho) devido ao solo apresentar boa drenagem. Neste agro-ecossistema foi desenvolvido o experimento na safra 2012-13, Regossolo abrupto (RegAb, Tabela 1) distante $500 \mathrm{~m}$ do rio.

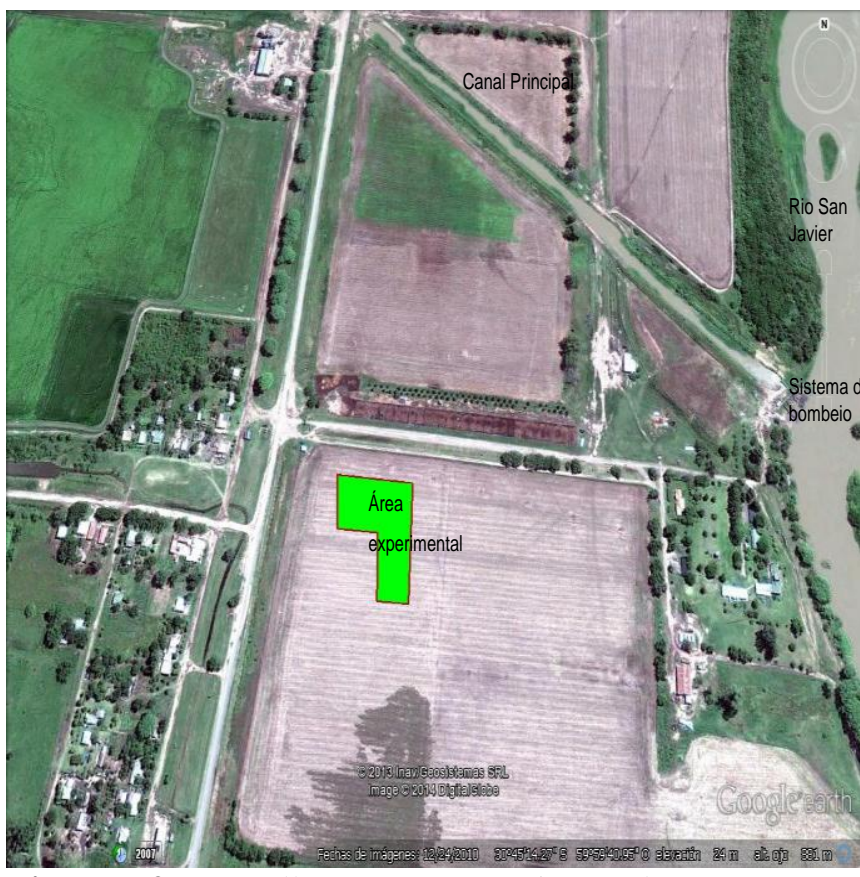

Figura 2. Detalhe área experimental no agroecossistema Terraço (Tç) com solo RegAb e lote sistematizado com declividade zero. Fonte: Google Earth, ingresso 10-01-14 
$\mathrm{Na}$ área dos experimentos foram realizadas as medições de infiltração com o método de duplos anéis para determinar a curva de velocidade de infiltração (VI) mediante a equação de Kostiakov. Para obter $\mathrm{Kh}_{\mathrm{s}}$ na camada superficial do solo foram utilizados tensioinfiltrômetros (PERROUX e WHITE, 1988), aplicando três tensões ( $\tau$ ): $0,1,5$ e $3 \mathrm{~cm}$ em sitios adjacentes para o cálculo da $\mathrm{Kh}_{\mathrm{s}}$. Essa sequência de medições foi realizada nas duas áreas experimentais e com quatro repetições.

As parcelas tinham dimensões aproximadas de $10 \mathrm{~m}$ x $10 \mathrm{~m}$ e cada uma foi circundada por taipas elevadas $0,25 \mathrm{~m}$, compactadas suficientemente para evitar vazamentos e fluxo debaixo das taipas (seepage) e isolar assim o manejo da água entre tratamentos. A construção destes bordos foi realizada com uma entaipadeira que tem um rolo lastrado que permite suficiente compactação. Entre parcelas manteve-se uma distância de aproximadamente $2 \mathrm{~m}$ sem plantas para condição de bordadura (BEECHER et al., 2006), que funcionaram também como carreadores. De uma valeta adjacente à parcela foi localizado um conjunto moto-bomba de acionamento manual que possibilitou a irrigação através de uma rede pressurizada.

No agro-ecossistema $\mathrm{Vz}$ a semeadura foi tradicional, com preparo do solo que consistiu em três passadas de grade de discos e uma passada de grade de dentes. No agro-ecossistema Tç foi utilizado o plantio direto, com semeadora pneumática. $\mathrm{O}$ arroz cultivado foi tipo índico, tropical-subtropical, grão longo fino (agulhinha), anaerçobico (ANA), com resistência a herbicida da família das imidazolinonas (clearfield CL). Em ambos os agro-ecossistemas foi utilizada a variedade Puitá-Inta, semeada em 11/11/11 e 13/10/12, com densidades de $100 \mathrm{~kg} \mathrm{ha}^{-1}$ e 120 $\mathrm{kg} \mathrm{ha}^{-1}$ respectivamente. Foram utilizados dois manejos da água: a) SIN, onde após de cada inundação (lâminas entre 3 a $8 \mathrm{~cm}$ ) dejou-se infiltrar a água até o nível do solo e iniciou-se novamente a irrigação, repondo à altura original da lâmina, mantendo assim o solo saturado; e b) NSA, no qual as irrigações foram feitas com aspersores quando as leituras dos tensiômetros de cápsula porosa $(0,2 \mathrm{~m})$ atingiam $-25 \mathrm{kPa}$ no solo PlaNa (STONE et al., 1986; STONE; SILVEIRA, 2004) e -10 kPa na profundidade de 0,1 m no RegAb. O critério de irrigação com aspersão foi a aplicação de uma lâmina fixa com frequência variável e o começo da irrigação foi igual em ambos os tratamentos. Uma pequena fração do nitrogênio foi aplicação na semeadura (20\%) na fileira e o restante como uréia (a lanço), no perfilhamento. Os macronutrientes pouco móveis $\left(\mathrm{P}\right.$ e $\left.\mathrm{K}^{+}\right)$foram aplicados na fileira junto com as sementes, em todos os tratamentos por igual. O delineamento estatístico adotado foi de parcelas divididas com repetições aleatórias (4), sendo os agro-ecossistemas a parcela principal, enquanto as sub-parcelas receberam os respectivos manejos da água, resultando assim em 16 parcelas.

O acompanhamento da fenologia em ambas safras foi feito segundo Counce et al. 
(2000) e com frequência semanal. Também foram determinadas as somas térmicas (ST) para a região de San Javier. A temperatura base $(\mathrm{Tb})$ adotada foi de $11^{\circ} \mathrm{C}$ (STEINMETZ et al., 2009). Os dados de temperatura máxima (Tmax), média (Tm) e mínima (Tmin) do ar $\left({ }^{\circ} \mathrm{C}\right)$ e radiação global (RG, W $\mathrm{m}^{-2}$ ) foram obtidos de uma estação meteorológica automática localizada nas áreas experimentais.

Ao final do ciclo foi feita a colheita manual das plantas, selecionando aleatoriamente quatro sitios das parcelas que totalizaram uma superfície de $1 \mathrm{~m}^{2}$. Os colmos foram cortados ao nível do solo e contadas as panículas, escolhendo aleatoriamente uma alíquota de cinquenta colmos com panícula para determinar os componentes da produtividade (WEBER et al., 2003; BOUMAN et al., 2006). Tanto os colmos da alíquota quanto ao resto da amostra foram trilhados manualmente, separando os grãos da palha. Os grãos do resto da amostra foram pesados em balança eletrônica e obtida sua umidade (\%) com umedímetro modelo TESMA Plus (TESMA s.a.i.c.). Uma fração da palha do resto foi pesada e secada em estufa a $65^{\circ} \mathrm{C}$ até massa constante para obter sua umidade. Os grãos e a palha da alíquota foram levados a estufa para secagem e após também foram pesados em balança eletrônica. Com estes resultados foram determinadas as seguintes componentes da produtividade: a) número de espiguetas vazias por panícula, separando-os mediante uma suave corrente de ar e contando manualmente; b) número de espiguetas cheias por panícula, contando manualmente os grãos afastados segundo procedimento anterior. Essas componentes foram expressas em percentagem com relação ao número de espiguetas totais e denominadas esterelidade (\%E) e fertilidade das espiguetas (FE) respectivamente, sendo testada a normalidade com o método de Shapiro-Wilks modificado (InfoStat, 2012); c) número de espiguetas totais (NEt) por panícula, somando os anteriores; d) massa de mil grãos (M1000), contando quatro grupos de 250 grãos e pesando com balança eletrônica de precisão; e) biomassa de grãos em casca $(\mathrm{BG})$ e da palha (BPa) e f) produtividade dos grãos (PG), corrigindo os valores de $\mathrm{BG}$ para $14 \%$ de umidade. A biomassa aérea total (BAT) foi obtida como a soma de BG mais $\mathrm{BPa}$, e também foi obtido o número de panículas por metro quadrado (NPan $\mathrm{m}^{-2}$ ) somando as panículas do resto e da alíquota. O índice de colheita (IC) foi obtido como a relação entre BG e BAT. Finalmente foram desenvolvidas correlações entre PG e componentes da produtividade utilizando o coeficiente de correlação linear de Pearson (pacote estatístico InfoStat, 2012).

\section{RESULTADOS E DISCUSSÃO}

Os dois solos são diferentes quanto a propriedades químicas, físicas e hidrológicas. No solo PlaNa, os valores de MO, CEe, $\mathrm{pH}, \mathrm{Mg}^{2+} \mathrm{e}$ $\mathrm{K}^{+}$obtidos na camada superficial são considerados ótimos para uma alta produtividade de 
arroz, no entanto, o $\mathrm{Ca}^{2+}$ e a PST estariam no limite inferior (Tabela 1). Já na camada subsuperficial, mais variáveis tiveram valores restritivos, fundamentalmente a PST, que se apresentou com o dobro do valor limite. A abundância do sódio é o que caracteriza estes solos, além de apresentar o $\mathrm{pH}$ acima do limiar ótimo (Tabela 1). $\mathrm{O} \mathrm{Ca}^{2+}$ e $\mathrm{Mg}^{2+}$ ficaram próximos ao limite inferior, indicando provável carência destes elementos, no entanto a disponibilidade do $\mathrm{K}^{+}$e $\mathrm{P}$ foram adequadas.

$\mathrm{O}$ solo RegAb não tem restrições para a cultura do arroz de alta produtividade na camada superficial (0 a 0,19 m, Tabela 1). Os teores de $\mathrm{Na}^{+}$são baixos, ao contrário do PlaNa. Quanto a MO, estaria levemente abaixo do limite recomendado, no entanto $\mathrm{P}$ teve magnitudes muito altas e maiores aos valores médios do agro-ecossistema Vz (Tabela 1). A relação $\mathrm{CO} / \mathrm{N}$ nas camadas superficial e sub-superficial foram muito similares aos do PlaNa, ainda que com menores teor de MO e Nt (Tabela 1).

O teste de infiltração com anéis duplos no solo PlanNa foi feito anteriormente ao inicio da irrigação (Tabela 2), no entanto, o teste com anéis duplos não foi possível se realizar no RegAB devido ao solo no interior do anéis expandiu-se e aumentou seu volume, selando os poros e impedindo que a água infiltrasse. Como alternativa foi avaliada a infiltração nas próprias parcelas submetidas a manejo com inundação, uma vez começada à irrigação. $\mathrm{O}$ procedimento foi aplicar, no final da tarde, uma lâmina de água e ler as réguas instaladas em cada parcela e, no amanhecer do dia seguinte, voltar a ler as réguas, resultando assim a diferença entre leituras a lâmina média infiltrada (Tabela 2). As diferenças entre os valores de VIB devem-se às diferentes metodologias utilizadas, sendo que a medição na própria parcela resultou a mais confiável. Quanto às medidas com infiltrômetro de tensão, elas resultaram em um alto $\mathrm{CV}$, produto da variabilidade espacial do solo (Tabela 2). $\mathrm{A} \mathrm{Kh}_{\mathrm{s}}$ do RegAb resultou no dobro da $\mathrm{Kh}_{\mathrm{s}}$ do PlaNa.

Tabela 2. Condutividade hidráulica nas tensões de $3 \mathrm{~cm}\left(\mathrm{Kh}_{3}\right), 1,5 \mathrm{~cm}\left(\mathrm{Kh}_{1,5}\right)$ e saturada $\left(\mathrm{Kh}_{\mathrm{s}}\right)$ e velocidade de infiltração básica (VIB) para os solos PlaNa e RegAb

\begin{tabular}{cccccc}
\hline Solo & $\begin{array}{c}\mathrm{Kh}_{3} \\
\left(\mathrm{~cm} \mathrm{~h}^{-1}\right)\end{array}$ & $\begin{array}{c}\mathrm{Kh}_{1,5} \\
\left(\mathrm{~cm} \mathrm{~h}^{-1}\right)\end{array}$ & $\begin{array}{c}\mathrm{Kh}_{\mathrm{s}} \\
\left(\mathrm{cm} \mathrm{h}^{-1}\right)\end{array}$ & $\begin{array}{c}\mathrm{VIB} \\
\left(\mathrm{cm} \mathrm{h}^{-1}\right)\end{array}$ & $\begin{array}{c}\mathrm{VIB} \\
\left(\mathrm{mm} \mathrm{dia}^{-1}\right)\end{array}$ \\
\hline PlaNa & 0,14 & 0,5 & 1,06 & $0,42(1)$ & 100,8 \\
$\mathrm{CV}(\%)$ & 13 & 35 & 32 & 25 & \\
RegAb & 0,43 & 0,68 & 2,33 & $0,12(2)$ & 28,8 \\
$\mathrm{CV}(\%)$ & 32 & 34 & 31 & 25 & \\
\hline
\end{tabular}

Nota - (1) Estimada segundo Grassi (1987) a partir de ensaio com duplo anéis; (2) Determinada nas parcelas com inundação

A ocorrência dos estádios ontogênicos foi igual em todos os tratamentos, a exceção do manejo NSA no agro-ecossistema Vz. A duração 
total do ciclo do arroz em ambas os agroecossistemas foi similar, embora as datas de semeadura tivessem quase 30 dias de diferença. No agro ecossistema $\mathrm{Vz}$ as plantas emergiram 12 dias após da semeadura (DAS) com uma densidade média de $181 \mathrm{pl} \mathrm{m}^{-2}$, no entanto, em Tç a emergência foi 11 DAS, com densidade média menor, de $132 \mathrm{pl} \mathrm{m}^{-2}$. A análise estatística indicou que não houve diferenças significativas $(\mathrm{P}<0,05)$ entre os tratamentos em nenhum dos agro ecossistemas (dados não apresentados). A diferença na densidade de plantas na emergência foi devido à palha da colheita anterior (plantio direito), já que a semeadora pneumática não conseguiu sua adequada ruptura e deixou nela muitas sementes. O ciclo da irrigação iniciou-se entre 4 a 5 folhas (V4-V5) indicando o inicio de perfilhamento, 22 e 25 dias após da emergência (DAE) nos agro ecossistemas $\mathrm{Vz}$ e $\mathrm{Tç}$ respectivamente. Os perfilhos foram identificados quando tenham 4 folhas (FAGERIA, 2007). O período vegetativo teve uma duração igual em ambas os agro ecossistemas, de 55 DAE (Tabela 3), o que representou aproximadamente a metade do ciclo total da cultura, próprio de cultivares de arroz ANA índicos semi-enanos (YOSHIDA, 1981). Porém, as somas térmicas (ST) para alcançar R1 foram menores no agro ecossistema Tç $(9 \%)$ devido à semeadura antecipada (Tabela 3).
Tabela 3. Estádios ontogênicos do cultivar Puitá INTA CL observados a campo e segundo Somas Térmicas (ST) totais, considerando dias após da semeadura (DAS), dias à emergência (DAE) e duração de cada estádio (DE), nos agroecossistemas várzea $(\mathrm{Vz})$ e terraço $(\mathrm{Tç})$

\begin{tabular}{|c|c|c|c|c|c|c|c|c|}
\hline \multirow{2}{*}{$\begin{array}{c}\text { Estádios } \\
\text { ontogênicos }\end{array}$} & \multicolumn{5}{|c|}{ Agroecossistema Vz } & \multicolumn{3}{|c|}{ Agroecossistema Tç } \\
\hline & $\begin{array}{l}\text { DAS } \\
\text { dias }\end{array}$ & $\begin{array}{l}\text { DAE } \\
\text { dias }\end{array}$ & $\begin{array}{c}\mathrm{ST} \\
{ }^{\circ} \mathrm{C} \text { dia }\end{array}$ & $\mathrm{DE}$ & $\begin{array}{l}\text { DAS } \\
\text { dias }\end{array}$ & $\begin{array}{l}\text { DAE } \\
\text { dias }\end{array}$ & $\begin{array}{c}\text { ST } \\
{ }^{\circ} \mathrm{C} \text { dia }\end{array}$ & $\begin{array}{l}\mathrm{DE} \\
\text { dias }\end{array}$ \\
\hline Emergência & 12 & - & - & 12 & 11 & - & - & 11 \\
\hline Emergência -R1 & 67 & 55 & 806 & 55 & 66 & 55 & 736 & 55 \\
\hline R1-R4 & 97 & 85 & 1.264 & 30 & 93 & 82 & 1.104 & 27 \\
\hline R4-R8 & 117 & 105 & 1.544 & 20 & 115 & 104 & 1.417 & 22 \\
\hline Colheita & 127 & - & - & 10 & 125 & - & - & 10 \\
\hline
\end{tabular}

No estádio R2 o manejo NSA (Vz) teve um atraso produto de um incipiente estresse hídrico. No estádio R4 da escala de Counce et al. (2000) e para o agro ecossistema Vz houve diferenças segundo o manejo da água. No tratamento SIN ocorreu 85 DAE e no NSA 6 dias após, indicando um atraso que foi mantido até a maturidade fisiológica. No agro ecossistema Tç não houve diferenças entre manejos, e o estádio R4 teve apenas 3 dias menos até a emergência que em Vz, embora a ST seja 16\% menor (Tabela 3). Ambos os agro ecossistemas tiveram similares DAE para alcançar R8, embora em Tç a duração do estádio (DE) entre R4 e R8 foi levemente maior (Tabela 3). 
Ao efeito de comparar as variáveis meteorológicas de maior influência no desenvolvimento de arroz ANA, isto é, temperaturas (máximas, mínimas e médias) e radiação global, entre San Javier e outras regiões com alta produtividade a campo $(8$ a 16,5 $\mathrm{Mg} \mathrm{ha}^{-1}$ ), foi elaborada a Tabela 4. A comparação permitiu inferir que San Javier apresenta valores de RG - acumulada e média-, Tm, Tmin e Tmax similares aos locais com maior produtividade de arroz do mundo indicando, portanto, que esses fatores permitiriam que esta Região também obtivesse altas produções de arroz irrigado.

Tabela 4. Radiação global (RG) total e média diária do ciclo, temperatura média (Tm), mínima (Tmin) e máxima (Tmax) para diferentes sítios do mundo com produção de arroz ANA

\begin{tabular}{|c|c|c|c|c|c|c|c|}
\hline Local & Latitude & $\begin{array}{l}\text { Altitude } \\
\text { (m) }\end{array}$ & $\begin{array}{c}\mathrm{RG} \\
\left(\mathrm{MJ} \mathrm{m}^{2}\right) \\
\text { total do } \\
\text { ciclo }\end{array}$ & $\begin{array}{c}\mathrm{RG} \\
\left(\mathrm{MJ} \mathrm{m}^{2}\right. \\
\left.\mathrm{dia}^{-1}\right) \\
\text { média } \\
\text { diária }\end{array}$ & $\begin{array}{r}\mathrm{Tm} \\
\left({ }^{\circ} \mathrm{C}\right)\end{array}$ & $\begin{array}{l}\text { Tmin } \\
\left({ }^{\circ} \mathrm{C}\right)\end{array}$ & $\begin{array}{c}\text { Tmax } \\
\left({ }^{\circ} \mathrm{C}\right)\end{array}$ \\
\hline $\begin{array}{l}\text { Argentina } \\
\text { (Santa Fe) }\end{array}$ & $30^{\circ} \mathrm{S}$ & 20 & 2.635 & 23,0 & 23,8 & 18,9 & 29,6 \\
\hline $\begin{array}{l}\text { Argentina } \\
(\mathrm{ER})(1)\end{array}$ & $31^{\circ} \mathrm{S}$ & 150 & 2.641 & 20,1 & 23,8 & - & - \\
\hline $\begin{array}{c}\text { China } \\
\text { (Yunnan) (2) }\end{array}$ & $26^{\circ} \mathrm{N}$ & 1170 & 2.380 & 17,2 & 24,7 & 24,0 & 30,1 \\
\hline $\begin{array}{c}\text { Japão } \\
\text { (Kyoto) (2) }\end{array}$ & $31^{\circ} \mathrm{N}$ & 20 & 1.817 & 13,2 & 23,8 & 19,9 & 28,4 \\
\hline $\begin{array}{c}\text { Filipinas } \\
\text { (Los Baños) } \\
\text { (3) }\end{array}$ & $14^{\circ} \mathrm{N}$ & 21 & 2.856 & 20,4 & 27,3 & 23,6 & 30,9 \\
\hline $\begin{array}{l}\text { Austrália } \\
(\mathrm{NSW})(4)\end{array}$ & $34^{\circ} \mathrm{S}$ & 140 & 3.233 & 23,0 & $\mathrm{~s} / \mathrm{d}$ & $\mathrm{s} / \mathrm{d}$ & $\mathrm{s} / \mathrm{d}$ \\
\hline $\begin{array}{c}\text { Brasil (RS) } \\
\text { (5) }\end{array}$ & $29^{\circ} \mathrm{S}$ & 170 & 3.300 & 22,0 & 24 & - & - \\
\hline
\end{tabular}

(1) Quintero (2009), (2) Katsura et al. (2008), (3) Bouman et al. (2006), (4) NSW- New South Wales, Humphreys et al. (2006), (5) Steinmetz et al. (2009); s/d sem dados.
Houve diferenças estatísticas $(\mathrm{P}<0,05)$ de PG entre os agro ecossistemas e entre os manejos da água (Tabela 5). A razão disto foi o manejo NSA no agro ecossistema $\mathrm{Vz}$, que apresentou deficiência hídrica no período reprodutivo e, decorrentemente, diminuição de $24 \%$ da produtividade (Figura 3 i). Porém, a aspersão no agro ecossistema Tç teve um desempenho satisfatório, sem diferenças estatísticas com SIN. A principal componente que explicou a menor produtividade no manejo NSA do agro ecossistema Vz foi o NPan $\mathrm{m}^{-2}$, que apresentou interação entre os agro-ecossistemas e os manejos da água (Figura 3 ii). Nessa Figura pode se observar a importante mortandade de colmos que ocorreu devido ao estresse hídrico no Vz, no entanto no Tç isso não aconteceu. A razão da maior mortandade seria a baixa redistribuição da água no perfil do solo que ocorreu no manejo NSA do agro-ecossistema Vz, sendo provável que os perfilhos mais jovens teriam raízes mais superficiais e pouco profundas, tornando-lhes vulneráveis em momentos de estresse hídrico (BOUMAN; TUONG, 2001). Embora hajam sido aplicadas lâminas de irrigação suficientes como para satisfazer os requerimentos das plantas, a água não estaria disponível para sua absorção pelas raízes destes perfilhos, agravado ainda mais pela elevada densidade do solo PlaNa $\left(1,63 \mathrm{Mg} \mathrm{m}^{-3}\right)$. De modo semelhante, na região de Texas (EEUU) somando a irrigação (aspersão) mais as PP, o arroz (variedade ANA) 
teve no total do ciclo entre 1.060 a $1.170 \mathrm{~mm}$, sendo estas quantidades $30 \%$ superiores a ETr, embora a cultura diminuiu sua produtividade em relação ao manejo com inundação continua (Mc CAULEY, 1990).

Tabela 5. Produtividade de arroz em casca (PG), massa de 1000 grãos (M1000), biomassa aérea total (BAT) e índice de colheita (IC) para os agro-ecossistemas $\mathrm{Vz}$ e Tç e os manejos SIN e NSA, San Javier, Argentina

\begin{tabular}{|c|c|c|c|c|c|c|c|c|}
\hline \multirow{2}{*}{ Componente } & \multicolumn{2}{|c|}{$\begin{array}{c}\text { Agro } \\
\text { ecossistemas }\end{array}$} & \multicolumn{2}{|c|}{ Manejos da água } & \multicolumn{4}{|c|}{ Fonte de variação } \\
\hline & $\mathrm{Vz}$ & $\mathrm{Tç}$ & SIN & NSA & PP & M & PPxM & $\mathrm{CV}$ \\
\hline $\begin{array}{c}\mathrm{PG} \\
\left(\mathrm{Mg} \mathrm{ha}^{-1}\right)\end{array}$ & $8,7 \mathrm{a}$ & $10,6 \mathrm{~b}$ & $10,4 \mathrm{~b}$ & $8,7 \mathrm{a}$ & * & $* *$ & ns & 15 \\
\hline $\begin{array}{c}\text { M1000 } \\
\left(\mathrm{g} 1000^{-1}\right)\end{array}$ & 23,0 & 23,8 & 23,3 & 23,3 & ns & ns & ns & 5 \\
\hline $\begin{array}{c}\text { BAT } \\
\left(\mathrm{Mg} \mathrm{ha}^{-1}\right)\end{array}$ & 18,5 & 21,3 & $20,8 \mathrm{~b}$ & $18,5 \mathrm{a}$ & ns & * & $\mathrm{ns}$ & 13 \\
\hline IC & 0,41 & 0,44 & 0,43 & 0,41 & ns & $\mathrm{ns}$ & ns & 10 \\
\hline
\end{tabular}

Nota - * significância $\mathrm{F}$ com $\mathrm{P}<0,05 ; * *$ significância $\mathrm{F}$ com $\mathrm{P}<0,01$; ns, não significativo

Tensões de água no solo em torno a $25 \mathrm{kPa}$ e profundidade de controle de $0,2 \mathrm{~m}$ não foram satisfatórias no solo PlaNa, no entanto $10 \mathrm{kPa}$ e profundidade de $0,10 \mathrm{~m}$ no solo RegAb permitiram adequada absorção de água. A grande diferença foi devido ao tipo de solo, já que no PlaNa as propriedades físicas limitaram a infiltração e movimentação das lâminas aplicadas. Stone et al. (1986) e Stone e Silveira (2004), propuseram uma profundidade de controle de 0,15 m e tensão de $25 \mathrm{kPa}$ para solos dos Cerrados com alta percolação, embora admitindo mermas da produtividade em torno a 10-15\%. O uso de tensiômetros de cápsula porosa nas camadas $0-0,1$ e $0,1-0,2$ no solo RegAb permitiram um adequado controle da irrigação e também definir o momento de reinício após de PP.

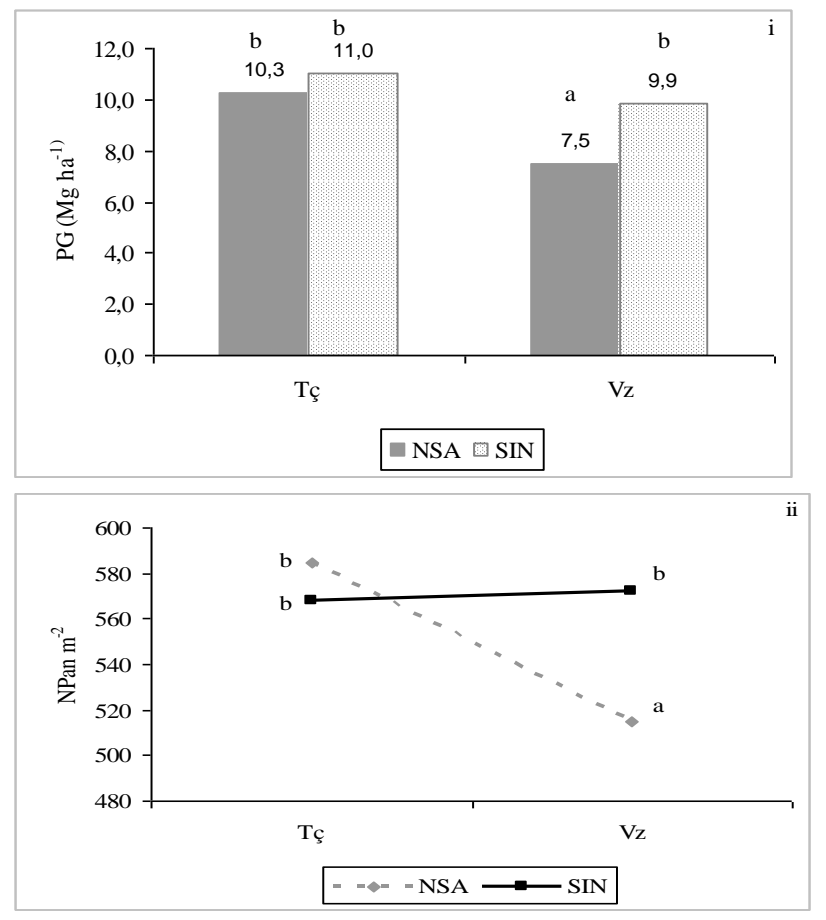

Figura 3. i) Produtividade de arroz em casca (PG) e ii) Número de panículas (NPan $\mathrm{m}^{-2}$ ) para os diferentes manejos da água (NSA e SIN) nos agroecossistemas Vz e Tç, San Javier, Argentina. Valores médios com letras diferentes indicam diferenças significativas $(\mathrm{P}<0,05)$ segundo teste LSD

A principal restrição que teve o manejo NSA no agro-ecossistema $\mathrm{Vz}$ foi à deficiência hídrica, que se manifestou em diferentes estádios fenológicos e órgãos do arroz. Esta deficiência deveu-se às baixas taxas de infiltração e condutividade hidráulica (Tabela 2). Pesquisas sob irrigação por aspersão na Argentina são poucas e a informação disponível é escassa. Westcott e Vines (1986) referem mermas na produtividade de arroz ANA devido a menor NEt com origem na deficiência hídrica no 
momento da diferenciação da panícula. Embora os autores indiquem que a umidade de solo foi adequada, considerando que a irrigação foi feita cada dois-três dias e o solo foi argiloso (Vertissolo), provavelmente o potencial de água no solo haja sido mais baixo que $-10 \mathrm{kPa}$ e haveria sido a razão do estresse hídrico. Mc Cauley (1990) também reporta diminuição da produtividade de arroz irrigado com aspersão (média de $20 \%$ em relação a Ic) devido a deficiência hídrica entre 32 e 5 dias prévios à floração, o que significou menor número de espiguetas por panícula. No Brasil as maiores quantidades de pesquisas com aspersão foram feitas em variedades de arroz anaeróbico (AE), que apresenta valores de FE mais baixos em condições de sequeiro, entre 60 a $70 \%$ (CRUSCIOL et al., 2003, ARTIGIANI et al., 2012) e aumentam significativamente com aspersão, ainda que não superam $80 \%$ (CRUSCIOL et al., 2006). No mesmo sentido, Toescher et al. (1997) determinaram perdas de produtividade (34\%) em cultivares de arroz ANA no Sul do Brasil, utilizando como controle da irrigação potenciais de água no solo de -33 $\mathrm{kPa}$. Isto indicaria que o problema do estresse hídrico deveu-se ao potencial do manejo da água antes que ao método de irrigação. Ao contrário, no agro-ecossistem Tç, esse manejo permitiu considerável economia de água, alcançando altas produtividades devido às melhor infiltração e condutividade hidráulica, que duplicaram os valores do PlaNa (Tabela 2).
A componente M1000 foi semelhante e sem diferenças estatísticas em todos os tratamentos (Tabela 5), indicando uma grande estabilidade nesta componente. A razão disso seria que o tamanho da espigueta é fortemente controlado pelo tamanho da casca, devido à genética de cada cultivar antes que às condições ambientais ou de manejo (YOSHIDA, 1981). No agro-ecossistema Tç houve uma leve tendência a menor massa no manejo NSA, ainda que não fosse devido à fonte, senão ao destino. Quintero (2009) determinou uma média de 23,4 g $1000^{-1}$ para o mesmo cultivar em Entre Ríos, Argentina (ER) muito semelhantes a San Javier.

A massa dos grãos da variedade Puitá INTA CL corresponde-se aos cultivares índicos, longos e finos, ainda que outras variedades ANA tenham maior $\mathrm{M} 1000$, até $28 \mathrm{~g} 1000^{-1}$ (QUINTERO, 2009) e variedades AE, também grão longo fino, de até $30 \mathrm{~g} 1000^{-1}$ (CRUSCIOL et al, 2006). Embora seja estável, a massa dos grãos teve resposta à irrigação na região dos Cerrados (Brasil), com veranico ou deficiência hídrica durante a floração e enchimento dos grãos, aumentando até $18 \%$. Weber et al. (2003) determinaram médias de 26,1 g $1000^{-1}$ para IRGA 417 irrigado, com muito baixa variação (CV de 3,5\%). Além disso, a menor duração do período de enchimento dos grãos da variedade Puitá INTA CL em San Javier, em relação à ER e o Sul do Brasil, não influiu na massa dos grãos, confirmando a estabilidade desta componente. Também corrobora isto a falta de correlação 
entre PG-M1000 em ambos os agro-ecossistemas (0,11 e 0,08 para Vz e Tç respectivamente).

Quanto a IC, também não houve diferenças, apesar do baixo coeficiente de variação desta componente (Tabela 5), e indicaria que a diminuição da produtividade no manejo NSA foi acompanhada com a mesma proporção de diminuição da BAT, sendo a deficiência hídrica responsável disso. No agroecossistema Tç os valores resultaram um pouco mais altos, resultando o manejo NSA menor a SIN (Tabela 5). Em ER a média de IC foi 0,52 para o cultivar Puitá INTA CL, maior aos obtidos em San Javier (QUINTERO, 2009).

A outra componente que teve diferença estatística $(\mathrm{P}<0,05)$ no manejo NSA com relação a SIN foi FE, no entanto NEt resultou semelhante (Figura 4). O cultivar Puitá INTA tem baixa quantidade de NEt, entre 70 e 100, ainda que alta FE em condições normais (QUINTERO, 2009). Esse autor refere, para ER, um valor médio de NEt de 96, no entanto Weber et al. (2003), para uma variedade similar (IRGA 417) cultivada no Sul do Brasil, uma média de 69. A componente NEt teve pouca variação em ambos os agro-ecossistemas, indicando que no período do enchimento dos grãos, o destino está influenciado mais por $\mathrm{N}^{\circ} \mathrm{Pan} \mathrm{m}^{-2}$ antes que pelo tamanho das panículas, que permanecem relativamente constantes embora as condições ambientais e de manejo sejam diferentes. Resultados similares relacionados à baixa variabilidade das espiguetas foram obtidos por Tao et al. (2006).
Quanto à esterilidade, Quintero (2009) refere para Puitá INTA (média de quatro safras) $11,9 \%$, sendo um dos cultivares com menor esterilidade. Esse valor foi semelhante ao obtido no tratamento SIN (Figura 4). A maior esterilidade das espiguetas no manejo NSA do agro-ecossistema $\mathrm{Vz}$ (16\%) não seria devido à radiação solar durante o período de enchimento dos grãos, já que RG nesse período foi adequada (294 MJ m²), senão ao estresse hídrico ocorrido prévio à floração e início de enchimento dos grãos (BOUMAN; TUONG, 2001). Yoshida (1981) refere que o estresse hídrico severo entre 3 a 11 dias prévios a floração pode provocar até o 60\% de esterilidade. Também não seria devido às condições não saturadas do solo, já que o desenvolvimento de arroz ANA nestas condições foi amplamente avaliada por Tao et al. (2006), encontrando que com manejo alternado de saturação/não saturação a principal componente que diminui a produtividade foi a quantidade de colmos férteis $\left(\mathrm{N}^{\circ} \mathrm{Pan}\right)$, no entanto a $\mathrm{FE}$ manteve-se estável com diferentes tratamentos de solo não saturado.

Dois problemas específicos do manejo com aspersão e relacionados com a cultura seriam a possibilidade de doenças e arraste dos grãos de pólen. Para evitar isto último, as irrigações -em geral para todo o ciclo, e durante a floração com maior cuidado-, começaram à 06:30, duravam média hora por bloco e culminavam entre $1,30 \mathrm{~h}$ e $2 \mathrm{~h}$ após, tratando de evitar o contato das gotas dos aspersores com os grãos de pólen. Para muitas variedades de arroz e dependendo das 
temperaturas, a antese ocorre entre as 08:00 e 13:00 (YOSHIDA, 1981). Porém, em alguns dias pôde haver superposição entre as aplicações e a antese, provocando possível arraste dos grãos de pólen.

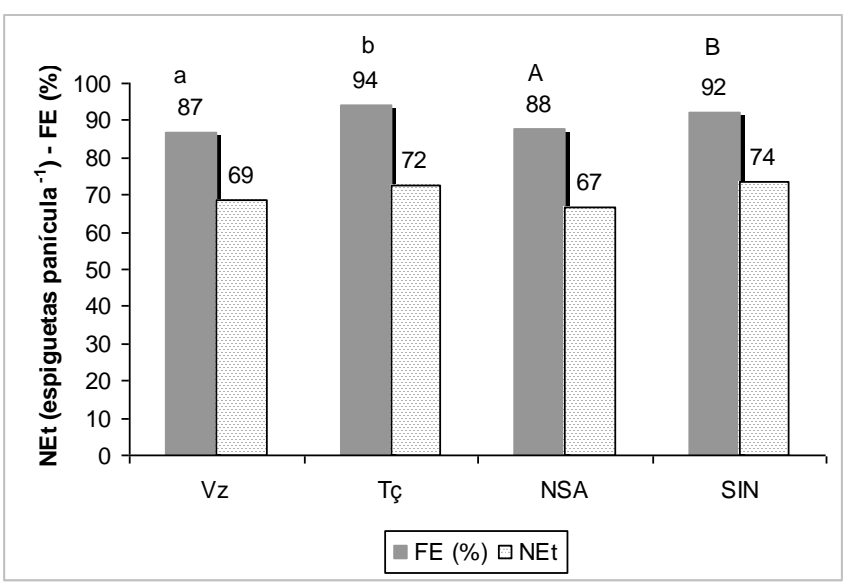

Figura 4. Número de espiguetas totais (NEt) e fertilidade de espiguetas (FE) para os manejos da água NSA e SIN nos agro-ecossistemas Vz e Tç. Valores médios de cada variável com letras diferentes maiúsculas nos manejos da água e minúsculas nos agro-ecossistemas, indicam diferenças significativas $(\mathrm{P}<0,05)$ segundo teste LSD

No agro ecossistema Tç foi apenas $3 \%$ maior que o manejo SIN e, na Vz, o impacto da deficiência hídrica na esterilidade das espiguetas haveria sido muito maior que o possível lavado dos grãos de pólen. Numa pesquisa com arroz irrigado com aspersão aplicou-se as doses de irrigação durante a tarde para evitar que as gotas dos aspersores tivessem impacto sob a flores abertas (Mc CAULEY, 1990). Porém, em condições reais a irrigação deve ser feita o dia inteiro para otimizar a equipe, sem possibilidades de suspender quatro ou cinco horas ao dia. Com tudo, a esterilidade de espiguetas foi a menor a $15 \%$ referido por
Yoshida (1981) para variedades ANA semi-anãs. Este mesmo autor refere que, em condições normais de fornecemento de água e nutrientes, a maior $\mathrm{N}^{\circ} \mathrm{Pan} \mathrm{m}^{-2}$ aumenta a esterilidade das espiguetas, ou diminui FE, devido a problemas para completar o enchimento. Isto não foi corroborado nenhum dos agro-ecossistemas, já que no Tç a correlação $\mathrm{N}^{\circ} \mathrm{Pan} \mathrm{m}^{-2}$ vs $\% \mathrm{E}$ foi não significativa $(0,2)$, no entanto no $\mathrm{Vz}$ ocorreu o contrário, isto é, houve correlação negativa altamente significativa $(-0,65)$, indicando que a mortandade de colmos e a esterelidade das espiguetas no NSA ocorreram em simultâneo. Os tratamentos com aspersão não foram afetados por doenças nenhum dos agro-ecossistemas, ainda na bibliografia há resultados contrastantes. Westcott e Vines (1986) referem diminuição na produtividade com aspersão devido à queima-dabainha (Rhizoctonia solani), com possível origem num aumento na umidade do dossel e molhado das folhas ou também por salpicadura das gotas com inóculos que se encontram nos restos vegetais. Porém, Marano et al. (2012), demonstraram que a aspersão com pivô central não incrementa nem a incidência nem a severidade de doenças na cultura de trigo. McCauley (1990) também relata que com aspersão as doenças em arroz ANA podem ser totalmente controladas.

Por outro lado, no agro-ecossistema Tç houve correlação positiva significativa entre NEt vs $\mathrm{N}^{\circ}$ Pan $\mathrm{m}^{-2}(0,48)$ no entanto no Vz também foi positiva, ainda que não significativa $(0,38)$. Isto 
contrasta com os resultados de Fageria (2007), quem refere que existe uma relação inversa entre o tamanho da panícula (NEt) e a quantidade de panículas per unidade de área $\left(\mathrm{N}^{\circ} \mathrm{Pan} \mathrm{m}^{-2}\right)$ devido a com maior número de panículas o destino também seria importante e haveria limitações na fonte. Segundo este suposto, em ambos os agro-ecossistemas não houve problemas de fonte para encher adequadamente os grãos. Uma provável razão dos resultados contrastantes seria que a variedade Puitá INTA tem panículas pequenas em relação às variedades AE avaliadas por Fageria (2007), com 150-200 espiguetas per panícula.

Os resultados obtidos nos experimentos foram muito diferentes às produtividades médias da Região, que no último quinquenio foi $6 \mathrm{Mg}$ $\mathrm{ha}^{-1}$, embora na safra 2012-13 de apenas 4,5 Mg $\mathrm{ha}^{-1}$. Essa diferença é superior à estimada por Quintero (2009) para trabalhos experimentais no $\mathrm{ER}$, sendo as possíveis razões que as aplicações de adubo, o controle das ervas daninhas e a colheita foram feitas manualmente, sem as perdas próprias das máquinas, além que o manejo da água foi certeiro e por isso as parcelas receberam maior atenção.

\section{CONCLUSÕES}

Em San Javier, Argentina, as condições de radiação e temperaturas são ótimas para obter altas produtividades de variedades de arroz
ANA. Nas condições do agro-ecossistema Vz o solo deve permanecer saturado durante todo o ciclo para o arroz (variedade ANA) se desenvolver sem restrições. $\mathrm{O}$ manejo intermitente satisfaz isso sem diminuir a produtividade, porquanto se apresenta como uma alternativa ao manejo de inundação tradicional, no entanto com aspersão as plantas tiveram deficiência hídrica que repercutiu na produtividade.

Para o agro-ecossistema Tç, o mesmo cultivar de arroz anaeróbico teve alta produtividade com solo não saturado, uma vez que o potencial de água no solo na camada 0$0,10 \mathrm{~m}$ fosse mantido em torno a $-10 \mathrm{kPa}$.

A dificuldade do manejo NSA no PlaNa deveu-se às baixas taxas de infiltração e condutividade hidráulica, que impediram a movimentação da água às camadas inferiores do solo. Ao contrário, no RegAb, obteve-se um desempenho muito satisfatório devido ao solo tem adequada infiltração e movimentação da água. Em função desta observação, a aspersão pode ser utilizada em variedades de arroz ANA em solos com boa drenagem que permitam infiltrar água até profundidade de 0,2-0,3 m.

\section{AGRADECIMENTOS}

Especial agradecimento aos engenheiros agrónomos Marcelo Peart, Rubén Favot, Noel 
Dopazo, Hernán Rougier, Jonatan Bianchi, Alfedo Payne, Salvador Muzzio, Juan Manual Murillo e alunos da graudação Martin Savino, Ulises Dopazo, Mariano Brondi, Diosnel Faccioli, Emiliano Bianchi, Gonzalo Scarpin e Emanuel Zucalli. Também agradecimento ao Governo do Estado de Santa Fe e da Universidad Nacional del Litoral pelos ajuda financeira para levar a cabo as pesquisas.

\section{REFERÊNCIAS BIBLIOGRÁFICAS}

ARTIGIANI, A. C. C. A.; CRUSCIOL, C. A. C.; ARF, O.; ALVAREZ R. C. F.; NASCENTE, A.S. Produtividade e qualidade industrial do arroz de terras altas em função da disponibilidade hídrica e adubação. Pesquisa Agropecuária Tropical, Goiânia, v.42, n.3, p.340-349, 2012.

\section{ASOCIACIÓN CORRENTINA DE} PLANTADORES DE ARROZ. Disponível em: $<$ http://www.acpaarrozcorrientes.org.ar/El_arroz _estadisticas/Estadistica_del_Arroz_en_Argentin a.> Acesso em: 16 dez. 2013.

BEECHER, H.G; DUNN B.W.; THOMPSON, J.A.; HUMPHREYS, E.; MATHEWS, S.K.; TIMSINA, J. Effect of raised beds, irrigation and nitrógeno management on growth, water use and yield of rice in south-eastern Australia. Australian Journal of Experimental Agriculture, Melbourne, v.46, n.1, p.13631372,July 2006.

BELDER, P.; BOUMAN B.A.M.; CABANGON, R.; GUOAN, L.; QUILANG, .J.P., LI, Y.; SPIERTZ, J.H.J.; TUONG, T.P.
Efect of water-saving irrigation on rice yield and water use in typical lowland conditions in Asia. Agricultural Water Management, Amsterdam, v.65, n.3, p.193-210,Mar. 2004.

BOUMAN, B.A.M.; TUONG, T.P. Field Water management to save water and increase its productivity in irrigated lowland rice. Agricultural Water Management, Amsterdam, v.49, n.1, p.11-30, July 2001.

BOUMAN, B.A.M.; XIAOGUANGB, Y.; HUAQIB, W.; ZHIMINB, W.; JUNFANGB, Z.; $\mathrm{BI}, \mathrm{C}$. Performance of aerobic rice varieties under irrigated conditions in North China. Field Crops Research, Amsterdam, v.97, n.1, p.5365, May 2006.

CARNEVALE, I; MARANO, R.P.; IMHOFF, S.; DAMIANO, F.F.; JÓBBAGY, E.G. Puede el Riego rehabilitar tierras para agricultura?. In: CONGRESO NACIONAL DEL AGUA, 30., 2011, Resistencia. Anais... Resistencia: INA, 2011. 1 CD-ROM.

COUNCE, P.A.; TERRY, C.K.; ANDREW, J.M. A uniform, objective, and adaptive system for expressing Rice development. Crop Science, Madison, v.40, n.2, p.436-443, Mar./Apr. 2000.

CRUSCIOL， C.A.C.; ARF，O.; SORATTO, R.P.; MACHADO, J.R. Influência de lâminas de água e adubação mineral na nutrição e produtividade de arroz de terras altas. Revista Brasileira de Ciência do solo, Campinas, v.27, n.4, p.647-657, 2003.

CRUSCIOL, C.A.C.; SORATTO, R.P.; ARF, O.; MATEUS, G.P. Yield of upland rice cultivars in rainfed and sprinkler-irrigated systemsin the Cerrado region of Brazil. Australian Journal of Experimental Agriculture, Melbourne, v.46, v.11, p.15151520, Oct. 2006. 
FAGERIA, N.K. Yield physiology of rice. Journal of Plant Nutrition, New York, v.30, n.6, p.843-879, June 2007.

FOOD AND
ORGANIZATION OF THE THE UNITED
NATIONS. Disponível em: <http://faostat.
fao.org/site/567/DesktopDefault.aspx?PageID=5
67\#ancor.> Acesso em: 16 dez. 2013 .

GRASSI C. Diseño y Operación del Riego por superfície. Caracas: CIDIAT, 1987. 415p.

HUMPHREYS, E.; LEWIN L.G.; KHAN, S.; BEECHER, H.G.; LACY, J.M.; THOMPSON, J.A.; BATTEN, G.D.; BROWN A.; RUSSELL, C.A.; CHRISTEN, E.W.; DUNN, B.W. Integration of approaches to increasing water use efficiency in rice-based systems in southeast Australia. Field Crops Research, Amsterdam, v.97, n.1, p.19-33, 2006.

INFOSTAT. Software estatístico. Cordoba, 2012. Disponível em: <http://www.infostat. com.ar/index.php>. Acesso em: 25 jan. 2014.

KATSURA, K; MAEDA S.; LUBIS, I.; HORIE, T.; CAO, W.; SHIRAIWA, T. The high yield of irrigated rice in Yunnan China: a cross-location analysis. Field Crops Research, Amsterdam, v.101, n.1, p.1-11, Apr. 2008.

MARANO, R.P.; FOLEGATTI M.V.; DOPAZO, N; ROUGIER, H. Avaliação da adubação com nitrogênio e manejos alternativos de água em arroz irrigado. In: CONGRESSO BRASILEIRO DE ENGENHARIA AGRÍCOLA, 42., Fortaleza, 2013. Anais... Fortaleza: SBEA, 2013. 1 CD-ROM.

McCAULEY, G.N. Sprinkler vs. flood irrigation in traditional rice production regions of southeast Texas. Agronomy Journal, Madison, v.82, n.4, p.677-83, Aug. 1990.
MEZZOMO, R.F. Irrigação contínua e intermitente em arroz irrigado: uso de água, eficiência agronômica e dissipação de imazethapyr, imazapic e fipronil. 2009. 61p. Dissertação (Mestrado em Agronomia) Universidade Federal de Santa Maria, Santa Maria, 2009.

MOSCONI, F.P; PRIANO, L.J.J.; HEIN, N.E.; MOSCATELLI, G.; SALAZAR, J.C.; GUTIÉRREZ, T.; CÁCERES, L. Mapa de suelos de la provincia de Santa Fe. Santa Fe: INTA-MAG, 1981.

PERROUX, K.M.; WHITE, I. Designs for disc permeameters. Soil Science Society of America Journal, Madison, v.52 n.5, p.1205-1215, 1988.

PILATTI, M.A.; D'ANGELO, C.H.; MARANO, R.P.; PENSIERO, J.F.; POTENTE H. LÓPEZ C.A. Ordenamiento territorial de la cuenca de Los Saladillos (Santa Fe). Santa Fe: UNL, 2003. 227p

PILATTI, M.A.; NORERO, A.L.; GRENÓN, Y D.A. Enfoque de sistemas y modelos de simulación de cultivos: Necesidad, formulaciones, usos $y$ evaluación. Madri: Editorial Académica Española, 2011. 140 p.

QUINTERO, C.; FIGUEROA, E.A. Fertilización del arroz. In: MELGAR R.; DÍAZ Z.M. Fertilización de cultivos y pasturas. Buenos Aires: INTA, 2008. chap. 3, p.156-189.

QUINTERO, C.E. Factores limitantes para el crecimiento y productividad del arroz en Entre Ríos, Argentina. 2009. 167p. Tese (Doutorado em Agronomia) - Universidad de Coruña, Coruña. 2009.

RAMONELL, C.G.; MARCHETTI, Z.Y.; BARBERIS I.M.; GIRAUDO, A.R.; 
BENZAQUÉN, L.; BLANCO, D.E.; BÓ, R.F.; KANDUS, P.; LINGUA, G.F.; MINOTTI, P.; QUINTANA, R.D.; SVERLIJ, S.; VIDAL, L.(Ed.) Inventario de los Humedales de Argentina. Sistemas de paisajes de humedales del Corredor Fluvial Paraná-Paraguay. Buenos Aires: Secretaria de Ambiente y Desarrollo Sustentable de la Nación, 2013. 380p.

STEINMETZ, S.; FAGUNDES, P.R.R.; MAGALHAES JUNIOR, A.M.; SCIVITTARO, W.B.; DEIBLER, A.N.; ULGUIM, A.R.; NOBRE, F.L.L.; PINTANEL, J.B.A.; OLIVEIRA, J.G.; SCHNEIDER, A.B. Soma térmica e número de dias para atingir os principais estádios de desenvolvimento de 16 cultivares de arroz irrigado. Pelotas: Embrapa Clima Temperado, 2009. 31p.

STONE, L.F.; MOREIRA, J.A.A.; SILVA, S.C. Tensão da água no solo e produtividade do arroz. Goiânia: EMBRAPA-CNPAF, 1986. 6p. (Comunicado Técnico).

STONE, L.F.; SILVEIRA, P.M. Arroz irrigado por aspersão. Informe Agropecuário, v.25, n.222, p.70-76, 2004.

TAO, H.; BRUECK, H.; DITTERT, K.; KREYE, C.; LIN, S.; SATTELMACHER B. Growth and yield formation of ricez (Oryza sativa L.) in the water-saving ground cover rice production system (GCRPS). Field Crops Research, Amsterdam, v.95, n.1, p.1-12, 2006.

TOESCHER, C.F.; RIGHES, A.A.; CARLESSO, R. Volume de água aplicada e produtividade do arroz sob diferentes métodos de irrigação. Revista da FZVA, Uruguaiana, v.4, n.1, p.75-79, 1997. Disponível em: $<$ http://revistaseletronicas.pucrs.br/ojs/index.php /fzva/article/viewArticle/1965.> Acesso em: 23 jan. 2014.

UNFPA. Estado da população mundial. New York, 2013. 100p.

WEBER, L.O.; MARCHEZAN, E.; CARLESSO, R.; MARZARI, V. Cultivares de arroz irrigado e nutrientes na água de drenagem em diferentes sistemas de cultivos. Ciência Rural, Santa Maria, v.33, n.1, p.27-33, fev. 2003.

WESTCOTT, M.P.; VINES, K.W.A. Comparison of sprinkler and flood irrigation for rice. Agronomy Journal, Madison, v.78, n.4, p.637-640, July 1986.

YOSHIDA, S. Fundamentals of rice crop science. Los Baños: IRRI, 1981. 279p. 